\title{
Construction et Validation d'une Échelle d'évaluation du Tribalisme dans les Relations Intergroupes
}

\author{
Messanga Gustave Adolphe, \\ Maître de Conférences de Psychologie Sociale, \\ Université de Dschang, Cameroun \\ Nzeuta Lontio Sylvestre, \\ Doctorant en Psychologie Sociale, \\ Université de Dschang, Cameroun
}

Doi:10.19044/esj.2020.v16n10p195 URL:http://dx.doi.org/10.19044/esj.2020.v16n10p195

\section{Résumé}

Le tribalisme est un phénomène social consistant en la tendance pour les individus à valoriser leur tribu au détriment des autres. Par conséquent, on peut le ranger dans la catégorie conceptuelle des discriminations intergroupes. À ce jour, la littérature psychométrique portant sur les discriminations ne comporte pas d'instrument permettant l'évaluation du traitement différencié des individus sur la base de leurs appartenances catégorielles tribales. Dans cette perspective, la présente étude se donne pour objectif de construire une échelle de mesure du tribalisme. Celle-ci a quatre dimensions relatives aux attitudes et comportements pro-endogroupes et anti-exogroupes. Elle est validée auprès de 890 participants des deux sexes, tous étudiants à l'Université de Dschang (Cameroun). Leur âge moyen est de 23.40 ans. Les Analyses Factorielles Exploratoires et en Composantes Principales des données collectées sont concluantes. L'indice alpha de l'échelle globale est conforme aux normes requises pour une méthode de mesure de cette nature $(\mathrm{N}=890 ; \alpha$ $\epsilon[.85 .86] ; \alpha=.859)$. Ces coordonnées métriques valident l'échelle.

Mots clés : Tribalisme, conscience tribale, discrimination intergroupe, Attitude pro-endogroupe et anti exogroupe, comportement pro-endogroupe et anti-exogroupe 


\title{
Construction and Validation of a Tribalism Assessment Scale in Intergroup Relations
}

\author{
Messanga Gustave Adolphe, \\ Maître de Conférences de Psychologie Sociale, \\ Université de Dschang, Cameroun \\ Nzeuta Lontio Sylvestre, \\ Doctorant en Psychologie Sociale, \\ Université de Dschang, Cameroun
}

\begin{abstract}
Tribalism is a social phenomenon consisting of the tendency for individuals to value their tribe at the expense of others. Consequently, it can be placed in the conceptual category of intergroup discrimination. To date, the psychometric literature on discrimination does not include an instrument allowing the evaluation of the differential treatment of individuals on the basis of their tribal categorical affiliations. In this perspective, the present study sets itself the objective of constructing a scale for measuring tribalism. This has four dimensions relating to pro-ingroup and anti-outgroup attitudes and behaviors. It has been validated with 890 participants of both sexes, all students at the University of Dschang (Cameroon). Their average age is 23.40 years. The Exploratory Factorial and Main Component Analyzes of the data collected are conclusive. The alpha index of the global scale conforms to the standards required for a measurement method of this nature $(\mathrm{N}=890 ; \alpha \in[.85$ $.86] ; \alpha=.859)$. These metric coordinates validate the scale.
\end{abstract}

Keywords: Tribalism, tribal awareness, intergroup discrimination, proingroup and anti-outgroup attitude, pro-ingroup and anti-outgroup behavior

\section{Introduction}

En Afrique subsaharienne, depuis l'accession des pays à la souveraineté internationale, les politiques ont souvent fait recours à une stratégie bien connue pour accéder aux positions hiérarchiques dominantes et s'y maintenir : diviser pour mieux régner. Concrètement, il s'agit d'opposer les groupes tribaux pour empêcher l'émergence d'une conscience citoyenne se situant au-delà des clivages générés par les marqueurs d'identité (Mbonda, 2003 ; Obami, n.d.). Le danger que représenterait cette conscience citoyenne est qu'elle est susceptible d'amener les individus à adopter des comportements 
politiques basés sur des intérêts sociaux, économiques ou politiques, plutôt que sur des liens filiaux avec les autorités; d'où la possibilité pour eux de ne pas tenir compte de leurs appartenances ethniques pour se positionner au cours des échéances politiques comme les élections ou les mouvements de protestation populaires. Or, comme le révèlent François et al. (2014 a et b), les dirigeants des pays d'Afrique subsaharienne préfèrent très souvent aller vers les potentiels électeurs de leurs différentes localités d'origine à l'approche des échéances électorales, dans le but de les convaincre de les maintenir au pouvoir. Pour atteindre cet objectif, ils activent les appartenances ethniques desdits électeurs. Ainsi, ils n'hésitent pas à pratiquer une « ethnicité de l'ombre », souvent en rupture avec le discours anti-ethnique officiel (Messanga, 2018). Ces manœuvres sont particulièrement utiles parce qu'elles leur assurent le soutien politique dont ils ont besoin pour empêcher l'alternance. Elles ont cependant pour conséquences l'exacerbation des conflits interethniques et l'émergence d'une discrimination qui caractérise bien une politique de «décompensation et d'affaiblissement de l'unité. » (Mbonda, 2003 : 13) Il en découle que lorsqu'on adresse la problématique de la discrimination intergroupe en Afrique subsaharienne, le phénomène du tribalisme est au centre des préoccupations; d'où l'intérêt pour la recherche de comprendre les mécanismes qui le génèrent et l'entretiennent. Dans cette perspective, il faut relever que l'un des principaux obstacles auxquels sont confrontés les travaux portant sur ce phénomène sociétal est l'absence d'un instrument permettant d'en faire une évaluation quantitative (Jacobson \& Deckard, 2012), alors que la littérature indique que cette exaltation de la conscience tribale est une expérience observable et mesurable (Kelly, n.d. ; Minkala-Ntadi, 2011 ; Richerson \& Boyd, 2001 ; Roubaud, 1994). Remédier à cette situation est l'objectif de la présente recherche. Pour y parvenir, elle procède à une clarification conceptuelle, comme le préconise le paradigme de Churchill (1979).

Le concept de tribalisme vient du mot «tribu » qui définit un groupe de personnes partageant « une origine, une langue et une culture communes et ayant conscience de son unité ou de sa personnalité par rapport aux autres. » (Lombard, 1969 : 71) Il se manifeste concrètement par la loyauté envers la tribu d'appartenance et respecte le principe de la congruence cognitive. En effet, l'une des caractéristiques de ce phénomène social est la tendance, pour l'individu, à rechercher des éléments positifs dans les comportements des membres de l'endogroupe, afin de justifier leur acceptation comme membres de son groupe, et une tendance à davantage rechercher des éléments négatifs dans les comportements des membres de l'exogroupe, afin de justifier leur non acceptation. Cet ostracisme portant sur la tribu se situe dans la même perspective que le racisme lorsqu'il atteint un pic, du fait des séparations qu'il crée, en faisant obstacle à toute forme d'intégration entre différentes 
populations. C'est pourquoi on le classe dans la même catégorie conceptuelle que la ségrégation (Mbouobouo, 2018). En effet, le tribalisme est une ségrégation ethnique courante résultant d'une manipulation dont les conséquences les plus notables sont le renforcement des frontières intergroupes, le cloisonnement des populations et la création de microsociétés (Kpotufe, 2014 ; Tsafak, 2002).

Sur le plan historique, on situe la genèse du tribalisme dans la période coloniale. Précisément, les Colons auraient procédé par une manipulation identitaire, transformant les identités sociales en identités tribales (N'DiminaMougala, 2012). Dans les faits, le pouvoir colonial a transposé un schéma de pensée étranger pour une reconstruction identitaire mettant en exergue des identités ethniques et tribales (Assam, 2014). C'est le début d'une " colonisation ayant inculqué aux Nègres la conscience de leurs différences ethniques. » (Tchatchoua, 2012 : 22) En suivant la thèse de Kpotufe (2014), on peut dire que le tribalisme est devenu un danger en Afrique à l'époque où les Colons ont balkanisé le continent, en créant à dessein des territoires artificiels. Les affrontements parfois sanglants et meurtriers entre les tribus des différents pays du continent en sont la conséquence. Au Rwanda par exemple, c'est précisément dans les années 1950 que le tribalisme a été déployé comme l'une des armes des colonialistes contre les mouvements nationalistes d'une part, et comme une politique consistant à exciter le chauvinisme ethnique d'autre part (Kougoum, 2009). Ils ont opposé les groupes ethniques Hutu et Tutsi en instaurant une distinction sociale qui faisait des Tutsis des «Seigneurs », tandis que les Hutus étaient relégués au rang d' « esclaves » ou de «nègres ». Plus tard, on a assisté à un renversement de cette structure sociale avec désormais des «braves» Hutus et des «méchants » Tutsis. Fondée sur cette distinction, la révolution Hutu de 1959 s'est avérée désavantageuse pour l'ethnie Tutsi, dont les membres ont été condamnés à l'exclusion, à l'exil ou à la mort (Mbonda, 2003). Dans la même veine, on peut citer le cas du Cameroun, pays où environ 250 groupes ethniques se partagent le territoire national. Cette diversité démographique remarquable produit un contexte favorable à des tensions intergroupes, dont l'une des sources se trouve dans la période coloniale, où les administrateurs français ont dressé les groupes ethniques les uns contre les autres (Tchatchoua, 2012). Ces propos du Maréchal Lyautey en sont la preuve : «s'il y a des mœurs et des coutumes, il y a aussi des haines et des rivalités qu'il faut démêler et utiliser à notre profit, en opposant les uns aux autres, en nous appuyant sur les unes pour mieux vaincre les autres. » (Ondoua, $2013: 120$ ) Les dirigeants africains actuels se situent dans la même perspective. En effet, la finalité des manipulations politiciennes des ethnies et des tribus auxquelles ils se livrent est la conquête et le maintien au pouvoir. Il en découle qu'au sein de nombreux pays, le tribalisme constitue une tare sociale, emboîtée dans le 
système de gouvernance et exploitée pour des intérêts proprement politisés (Kougoum, 2009 ; Messanga, 2018). Il se déploie comme un moyen de manipulation des appartenances ethniques visant à attiser la haine tribale, qui se manifeste sous la forme d'une discrimination intergroupe.

Dans sa formulation initiale, la théorie de l'identité sociale s'inscrit dans la perspective des conflits intergroupes. Elle propose que les individus segmentent, classent et ordonnent leur environnement physique et social en catégories fondées sur la similitude entre les sujets qui les constituent (Autin, n.d. ; Licata, 2007). La conséquence de ce processus est que les individus se définissent comme membres de groupes au sein de la structure sociale (Fokou Dchoune et al., 2012). Dans les faits, la seule catégorisation des individus en deux groupes distincts entraîne la discrimination à l'encontre de l'exogroupe, pour différencier positivement l'endogroupe. Mankou (2007) parle de catégorisation identitaire pour faire référence à une forme de distinction intergroupe. Cette catégorisation est un outil de manipulation politique de l'ethnicité, pour créer une forme de discrimination intergroupe, conquérir le pouvoir et s'y maintenir. La catégorisation ethnique se caractérise par un référent mobilisé, qui place une personne dans une catégorie selon son identité fondamentale, relative à son origine ethnique (Barth, 1995).

Dhume (2011) soutient que la discrimination et l'ethnicisation s'imbriquent, non pas comme des exceptions dérogatoires à la norme, mais comme des formes de régulation parmi tant d'autres, prenant leur sens dans le fonctionnement banal des institutions. Dans cette perspective, la discrimination est un mécanisme d'assignation qui se base sur un processus de catégorisation. Elle permet d'établir une différence psychologique entre les groupes. La finalité de cette différenciation est d'acquérir une identité collective positive (Autin, n.d.). L'une de ses conséquences est la discrimination tribale (Mankou, 2007). En effet, l'ethnicité donne sens à l'explication des processus de discrimination tribale. Elle « consiste à priver quelqu'un de quelque chose à quoi il aurait droit, et d'abord à le priver d'égalité, sur la base de son appartenance à un groupe vu comme différent à raison de son origine supposée. » (Lorcerie, 2003 : 7). Cette discrimination se manifeste par le repli sur soi et la non-acceptation de l'étranger. « La tribu reste ici pour l'homme la limite aussi bien en face de l'étranger que vis-à-vis de soi-même. L'individu situe au centre de «l'univers » sa propre tribu à laquelle il attribue les grandes potentialités dans toutes les sphères de l'activité sociale. » (Mampouya, 1983 : 36) Ainsi, les individus centralisent leur tribu avec exagération et idéalisme; d'où le sentiment de supériorité de la tribu d'appartenance. En revanche, tout ce qui est hors de l'univers tribal est excentré ou rejeté. En ce sens, le tribalisme résulte d'une comparaison intergroupe favorable aux membres de l'endogroupe et défavorable aux membres des exogroupes; d'où le fait qu'il se manifeste par une forte 
tendance au sociocentrisme évaluatif (Licata, 2007). Ses conséquences attitudinales et comportementales sont l'endofavoritisme et l'exodéfavoritisme.

La littérature propose quelques instruments permettant d'évaluer le tribalisme. Cependant, ceux-ci présentent des limites importantes quand on les analyse dans une perspective psychométrique. Ainsi, Tsafak (2002) a conçu une échelle à quatre items qui, selon lui, évalue le tribalisme ou l'ethnocentrisme chez les élèves. Cet instrument est une échelle unidimensionnelle non standardisée, construite sur la base de quatre indicateurs de la distance sociale, dont le fait de partager un banc, une chambre, un groupe d'étude et une vie commune. À titre illustratif, on peut citer les deux items ci-après : si vous aviez la possibilité de choisir, accepteriez-vous de partager : a) le banc avec un camarade d'une autre ethnie ou tribu ; b) la chambre avec un camarade d'une autre ethnie ou tribu (Tsafak, 2002 : 221). En fait, ces items montrent clairement que cette échelle n'évalue pas réellement le tribalisme, mais plutôt la distance sociale. En effet, on peut davantage la rapprocher de l'échelle de distance sociale de Bogardus (1925) que d'une échelle évaluant la tendance à la discrimination basée sur les appartenances catégorielles, à l'image de l'échelle de racisme symbolique d'Henry et Sears (2002). Il est important de rappeler que le tribalisme repose sur une stéréotypisation qui augmente la distance sociale entre les groupes d'appartenance et de non appartenance des individus. Par conséquent, tout comme pour le nationalisme et le racisme, la distance sociale et l'ethnocentrisme peuvent simplement être considérés comme ses excroissances (Berting, 2001).

Le Tribalism Index de Jacobson et Deckard (2012) peut être considéré comme une méthode d'évaluation du tribalisme. Cet indice prend en compte les phénomènes caractéristiques des sociétés tribales. Il s'agit précisément des inégalités entre les sexes, de la perception de la corruption, des griefs et des fractionnements ethniques et linguistiques. Cet indice est donné par les pondérations suivantes : Tribalism Index $=$ Corruption Measure +0.5 (Ethnic Fractionalism $)+0.5$ (Indigenous Population $)+2$ (Gender Equality) + Group Grievance (Jacobson \& Deckard, 2012 : 10). Selon ses concepteurs, cet indice varie entre 1 et 160 . Cette variation correspond à un système de classement allant du plus haut niveau de tribalisme (avec un indice $=1$ correspondant à la première position dans le classement des pays tribalistes) au plus bas niveau de tribalisme (avec un indice $=160$ correspondant à la $160^{\text {ème }}$ position dans ce classement). La critique qu'on peut faire à cette méthode est qu'elle ne permet pas une évaluation directe du tribalisme, car l'indice auquel elle aboutit est le résultat de la pondération de plusieurs autres phénomènes sociaux, dont le tribalisme lui-même. Il en découle que les données collectées via cette méthode sont sujettes à caution. Même dans le cas où elle permettrait d'obtenir 
un indice du tribalisme, son niveau de fiabilité et de validité est questionnable. En effet, elle ne propose pas des items auxquels les individus répondraient et dont les scores permettraient d'établir ses coordonnées métriques, comme le stipule le paradigme de Churchill (1979). Il importe donc de proposer une échelle de mesure du tribalisme dans les relations intergroupes qui présente ces coordonnées.

Les méthodes d'évaluation du tribalisme relevées ci-dessus ne sont pas adaptées pour évaluer ce construit dans la perspective envisagée dans la présente étude. Pour rappel, son acception retenue ici en fait un phénomène caractérisé par des attitudes et des comportements discriminatoires, favorables aux groupes d'appartenance et défavorables aux groupes de non appartenance. Il est donc nécessaire de construire un instrument permettant d'appréhender ces aspects. Jacobson et Deckard (2012) suggèrent que la mise sur pied d'une telle méthodologie passe par la détermination des conditions optimales ou des réalités sociales voire politiques qui justifient ce phénomène, répertorient ses manifestations et présentent ses caractéristiques. Ces réalités ont été relevées dans les pages précédentes. Elles portent notamment sur les manipulations politiciennes des appartenances catégorielles tribales. Dans cette perspective, la présente étude procède à la construction et à la validation d'un instrument psychométrique standardisé permettant de quantifier la tendance à la discrimination tribale suivant d'une part, les attitudes et les comportements pro-endogroupes (favorables au groupe tribal d'appartenance), et d'autre part les attitudes et les comportements anti-exogroupes (défavorables aux groupes tribaux de non appartenance).

\section{Méthode}

\section{A. Participants :}

Les participants sont 890 étudiants des deux sexes (449 hommes et 441 femmes) inscrits dans diverses facultés de l'Université de Dschang. Leur âge moyen est de 23.40 ans. Ils sont originaires de différents groupes ethniques des dix régions du Cameroun et appartiennent à différentes classes sociales. Leur langue d'expression est le français. Ils ont tous marqué leur accord pour participer bénévolement à l'étude. Des garanties d'anonymat et de confidentialité leur ont été données quant à l'utilisation des informations qu'ils fourniraient pour cette recherche.

\section{B. Matériel et procédure :}

La recherche ne propose pas d'instrument qui évalue le tribalisme dans la perspective des discriminations intergroupes et présente des propriétés psychométriques, comme il en existe pour l'antisémitisme, le racisme, ou le sexisme. C'est ce à quoi remédie la présente étude, en développant une échelle permettant d'appréhender ce phénomène social comme une discrimination 
intergroupe basée sur les appartenances tribales. La discrimination intergroupe se manifeste généralement par les tendances à l'endofavoritisme et à l'exodéfavoritisme autant sur le plan attitudinal que comportemental.

Le processus de construction de l'échelle proposée dans la présente étude a consisté tout d'abord à générer les items sur la base la littérature portant sur la discrimination intergroupe en général et sur le tribalisme en particulier. Ensuite, on a procédé à une première collecte des données auprès des participants, comme le stipule la troisième étape du paradigme classique de Churchill (1979). Ce premier échantillon était constitué de 50 individus originaires de différents groupes ethniques du Cameroun. Cette première collecte avait pour objectifs de déceler les différents problèmes de compréhension que les items pouvaient poser aux participants et de recueillir leurs avis sur la nécessité de les reformuler. Bien que cette première phase de validation des items se soit avérée concluante, l'avis d'un expert dans le domaine a été sollicité. À l'issue du processus de construction, l'échelle psychométrique conçue dans cette recherche présente quatre (4) dimensions relatives aux attitudes et comportements pro-endogroupes (dimensions 1 et 2) et anti-exogroupes (dimensions 3 et 4). Ces dimensions qui constituent l'échelle globale sont évaluées par trente-six (36) items au total. Ces items n'évoquent ni un pays, ni une tribu, afin que l'échelle puisse être administrée en l'état, quels que soient les pays ou les entités tribales concernés par les recherches qui y auront recours.

La première dimension de l'échelle, relative aux attitudes proendogroupes (APEn), permet de déterminer si les individus ont des attitudes favorables à leur tribu d'appartenance. Elle comporte huit (8) items, dont le premier est formulé comme suit : «Dans la gestion de ce pays, les problèmes de ma tribu devraient d'abord être résolus avant ceux des autres tribus. ». La deuxième, portant sur les comportements pro-endogroupes (CPEn), comporte dix (10) items. Elle permet de savoir si les comportements des individus sont favorables à leur tribu d'appartenance. Par exemple, l'item 6 propose que : « Lors des élections, je vote un candidat en raison du fait qu'il appartient à ma tribu. ». La troisième, quant à elle, explore, avec huit (8) items, les attitudes anti-exogroupes (AAEx). Elle détermine à quel point les individus ont des attitudes défavorables à l'égard des autres tribus. À titre illustratif, l'item 3 de cette dimension postule que : «Les membres des autres tribus ne devraient pas être les plus promus de ce pays. ». La quatrième, qui évalue les comportements anti-exogroupes (CAEx) avec dix (10) items, permet de savoir à quel point les individus adoptent des comportements défavorables ou discriminatoires à l'encontre des membres des autres tribus. Par exemple, l'item 8 propose que : «Je m’oppose à la gouvernance de ce pays par des personnes autres que les membres de ma tribu. » 
La tâche des participants consiste à donner leur opinion sur chacun des items que comporte cet instrument de mesure, sur une échelle de Likert en sept (7) points, allant de 1 (Fortement en désaccord) à 7 (Fortement en accord). Le nombre de points qu'ils obtiennent permet de déterminer leurs tendances à la discrimination sur la dimension évaluée. Plus le score d'un participant est élevé dans une dimension, plus on considère qu'il a une tendance attitudinale ou comportementale endofavorable (dimensions 1 et 2 ) ou exodéfavorable (dimensions 3 et 4 ).

\section{Résultats}

Les résultats de la présente recherche s'intéressent uniquement à la structure interne de l'échelle du tribalisme. Cela permet de définir ses paramètres métriques, comme prévoit la théorie des instruments de mesure psychométriques. Premièrement, ces paramètres consistent en la détermination des indices d'adéquation de l'échantillon voire des contributions factorielles. Ces indices vérifient si les données obtenues sont quantitatives et forment un ensemble suffisamment cohérent et si les variables de l'échelle peuvent être regroupées en des facteurs latents ou en des composantes principales (Durand, 2005; Evrard et al., 2000). La détermination des coordonnées factorielles de l'échelle est envisagée. Le but de ces coordonnées est d'épurer statistiquement l'instrument d'une part et d'autre part d'estimer les inerties cumulées croissantes (variances totales expliquées) dudit instrument, afin de vérifier les quantités d'informations qu'il est susceptible de recueillir. La réalisation des graphiques factoriels des points-variables de la structure interne de l'échelle est effectuée dans le but de représenter graphiquement l'homogénéité interne de l'échelle. Deuxièmement, la méthode d'alpha de Cronbach est appliquée dans le but de déterminer les indices de cohérence interne des sous-échelles et de l'échelle globale. Ces indices alpha rendent compte de la fiabilité et la validité statistique de l'échelle développée (Bernaud, 2014). Pour assurer les liaisons internes de cette échelle, les indices d'association linéaire sont calculés, elles permettent de voir à quel degré les quatre sous-échelles du tribalisme sont liées entre elles d'une part et d'autre part à quel degré elles sont liées à l'échelle globale. Pour assurer la validité discriminante, une analyse de la variance est effectuée afin de mettre en évidence des différences entre des groupes de répondants pour lesquels des attitudes et des comportements tribalistes différents sont attendus. 


\section{A. Analyse des contributions factorielles et des variances totales expliquées en axes principaux d'inertie}

Tableau 1: Présentation des indices de KMO et des valeurs du test de sphéricité de Bartlett, des variances totales expliquées et des composantes principales $(\mathrm{CP})$ de l'échelle du tribalisme

\begin{tabular}{|c|c|c|c|c|c|c|c|c|c|c|c|c|}
\hline \multirow{2}{*}{\multicolumn{4}{|c|}{ Test de sphéricité de Bartlett }} & \multicolumn{9}{|c|}{ Valeurs propres initiales $(\lambda \alpha)$} \\
\hline \multirow[b]{2}{*}{ Échelle } & \multirow[b]{2}{*}{$\begin{array}{l}\text { Indice } \\
\text { KMO }\end{array}$} & & \multirow[b]{2}{*}{ Sig. } & \multicolumn{4}{|c|}{ Échelle globale } & \multicolumn{5}{|c|}{ Dimensions de l'échelle } \\
\hline & & $\begin{array}{c}\text { Khi } 2 \\
\text { approx. }\end{array}$ & & $\mathbf{C P}$ & Total & $\begin{array}{c}\% \\
\text { Variance }\end{array}$ & $\begin{array}{c}\% \\
\text { cumulé }\end{array}$ & & $\mathbf{C P}$ & Total & $\begin{array}{c}\% \\
\text { variance }\end{array}$ & $\begin{array}{c}\% \\
\text { cumulé }\end{array}$ \\
\hline \multirow{2}{*}{$\begin{array}{c}\text { Tribalis } \\
\text { me } \\
A P E n\end{array}$} & ,839 & $\begin{array}{c}8028,76 \\
7\end{array}$ & ,000 & 1 & 6,841 & 19,002 & 19,002 & \multirow[t]{2}{*}{$\begin{array}{c}A P E \\
n\end{array}$} & 1 & 2,697 & 33,714 & 33,714 \\
\hline & ,735 & $\begin{array}{c}1320,50 \\
0\end{array}$ & ,000 & 2 & 3,064 & 8,510 & 27,512 & & 2 & 1,291 & 16,133 & 49,847 \\
\hline CPEn & .761 & $\begin{array}{c}1532.13 \\
7\end{array}$ & .000 & 3 & 1,869 & 5,192 & 32,705 & \multirow{3}{*}{$\begin{array}{c}C P E \\
n\end{array}$} & 1 & 2,955 & 29,545 & 29,545 \\
\hline$A A E x$ & ,788 & $\begin{array}{c}1250,55 \\
6\end{array}$ & ,000 & 4 & 1,493 & 4,147 & 36,852 & & 2 & 1,364 & 13,636 & 43,182 \\
\hline \multirow[t]{7}{*}{ CAEX } & .734 & 942.816 & .000 & 5 & 1,372 & 3,811 & 40,663 & & 3 & 1,123 & 11,229 & 54,411 \\
\hline & & & & 6 & 1,285 & 3,570 & 44,232 & \multirow{3}{*}{$\begin{array}{c}A A E \\
x\end{array}$} & 1 & 2,500 & 31,253 & 31,253 \\
\hline & & & & 7 & 1,186 & 3,294 & 47,526 & & 2 & 1,088 & 13,600 & 44,853 \\
\hline & & & & 8 & 1,138 & 3,160 & 50,687 & & 3 & 1,012 & 12,654 & 57,506 \\
\hline & & & & 9 & 1,063 & 2,954 & 53,641 & \multirow{3}{*}{$\begin{array}{c}C A E \\
x\end{array}$} & 1 & 2,953 & 29,534 & 29,534 \\
\hline & & & & 10 & 1,011 & 2,807 & 56,448 & & 2 & 1,072 & 10,719 & 40,252 \\
\hline & & & & & & & & & 3 & 1,005 & 10,051 & $\mathbf{5 0 , 3 0 4}$ \\
\hline
\end{tabular}

Ce tableau présente premièrement des indices d'adéquation de l'échantillon de Kaiser-Meyer-Olkin (KMO) qui attestent que les corrélations partielles entre les variables sont fortes (Échelle globale $\mathrm{KMO}=.839, p<.05$; $\mathrm{APEn}, \mathrm{KMO}=.735, p<.05 ; \mathrm{CPEn}, \mathrm{KMO}=.761, p<.05 ; \mathrm{AAEx}, \mathrm{KMO}=$ $.788, p<.05 ;$ CAEx, KMO $=.734, p<.05)$. Le test de sphéricité de Bartlett est significatif $(p<.05)$ et indique que le modèle de facteur est adapté à cette échelle, car les poids factoriels observés vérifient la capacité des données à être factorisées. Deuxièmement, l'application des AFE et ACP révèle dix (10) facteurs principaux $\left(\mathrm{F}_{1} \times \mathrm{F}_{2} \times \ldots \times \mathrm{F}_{10}\right)$ ayant chacun une valeur initiale propre supérieure à 1 . Les pourcentages d'inertie cumulée récapitulés révèlent que cette échelle peut recueillir au moins $56,448 \%$ des informations sur le phénomène du tribalisme. Pour ce qui concerne les dimensions, on relève que l'APEn comporte deux (2) facteurs principaux $\left(\mathrm{F}_{1} \times \mathrm{F}_{2}\right)$ avec $49,847 \%$ des informations sur cette dimension. Les dimensions CPEn, AAEx et CAEx comportent chacune trois (3) facteurs principaux $\left(\mathrm{F}_{1} \times \mathrm{F}_{2} \times \mathrm{F}_{3}\right)$. Ils peuvent révéler chacun respectivement près de $54,411 \%, 57,506 \%$ et $50,304 \%$ des informations relatives à ces dimensions. Tous ces indices montrent que l'instrument développé a une structure interne satisfaisante. Une 
représentation graphique de ladite structure révèle la dimensionnalité de cette échelle et la disposition de ses éléments.

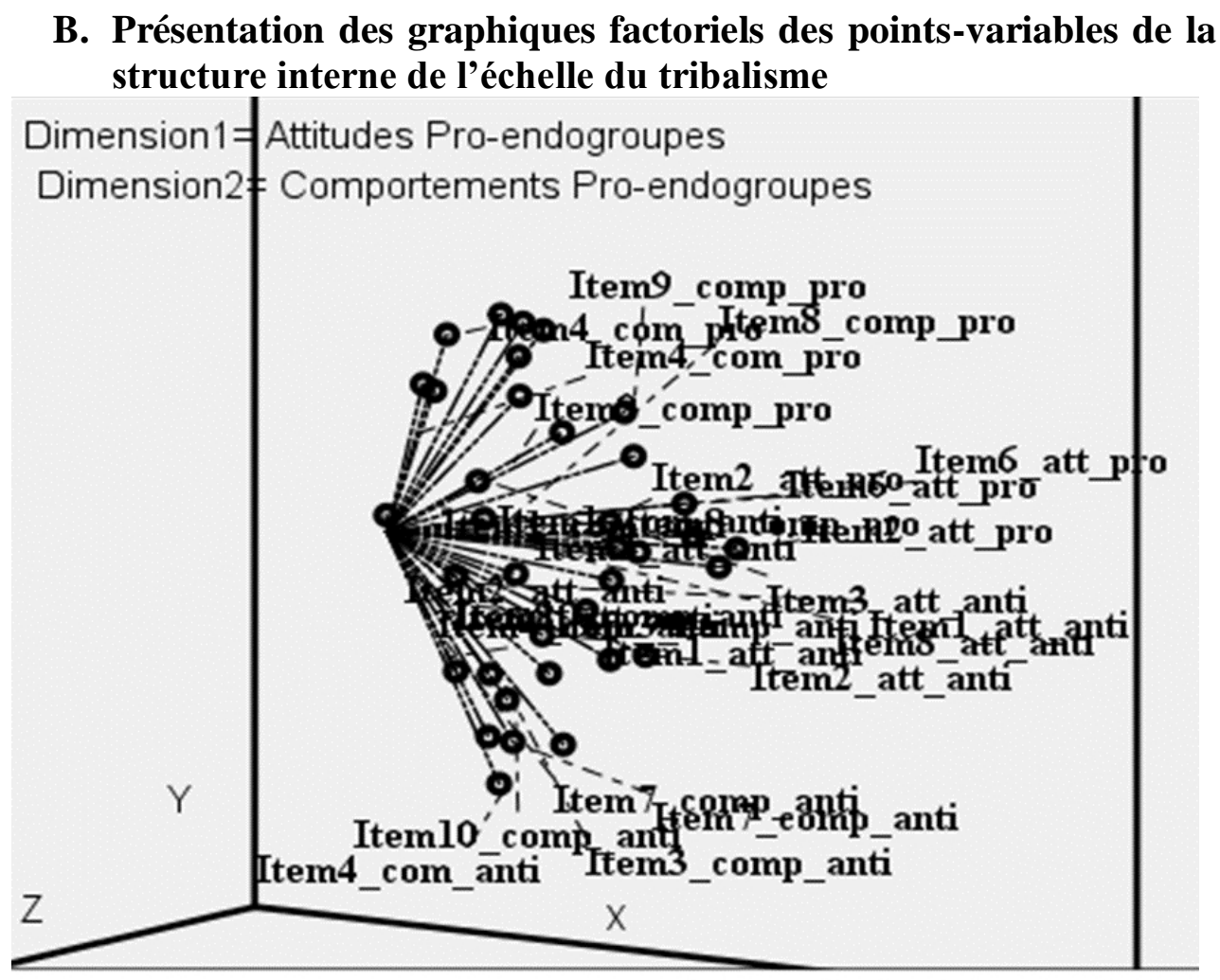

Dimension3= Attitudes Anti-e)

Figure 1: Carte factorielle de l'échelle du tribalisme

Cette figure représente la structure factorielle de l'échelle du tribalisme évaluée principalement avec dix (10) facteurs. Pour simplifier l'interprétation desdits facteurs, la méthode de rotation orthogonale Varimax a permis de minimiser le nombre de variables ayant de forts chargements sur chaque facteur. On voit un tracé en quatre dimensions des chargements de trois premiers facteurs sur les dix qui constituent l'échelle développée. En partant de la partie inférieure vers la partie supérieure du graphique factoriel des points-variables, on observe un groupement d'items relatifs aux CAEx, suivi au centre des items liés aux AAEx. On y observe un groupement d'items portant sur les APEn. Au-dessus de ceux-ci se trouvent les items évaluant les CPEx. Cette représentation en arbre à quatre banches émane d'une projection orthogonale et constitue la représentation exacte des quatre dimensions du tribalisme envisagée dans cette étude. 


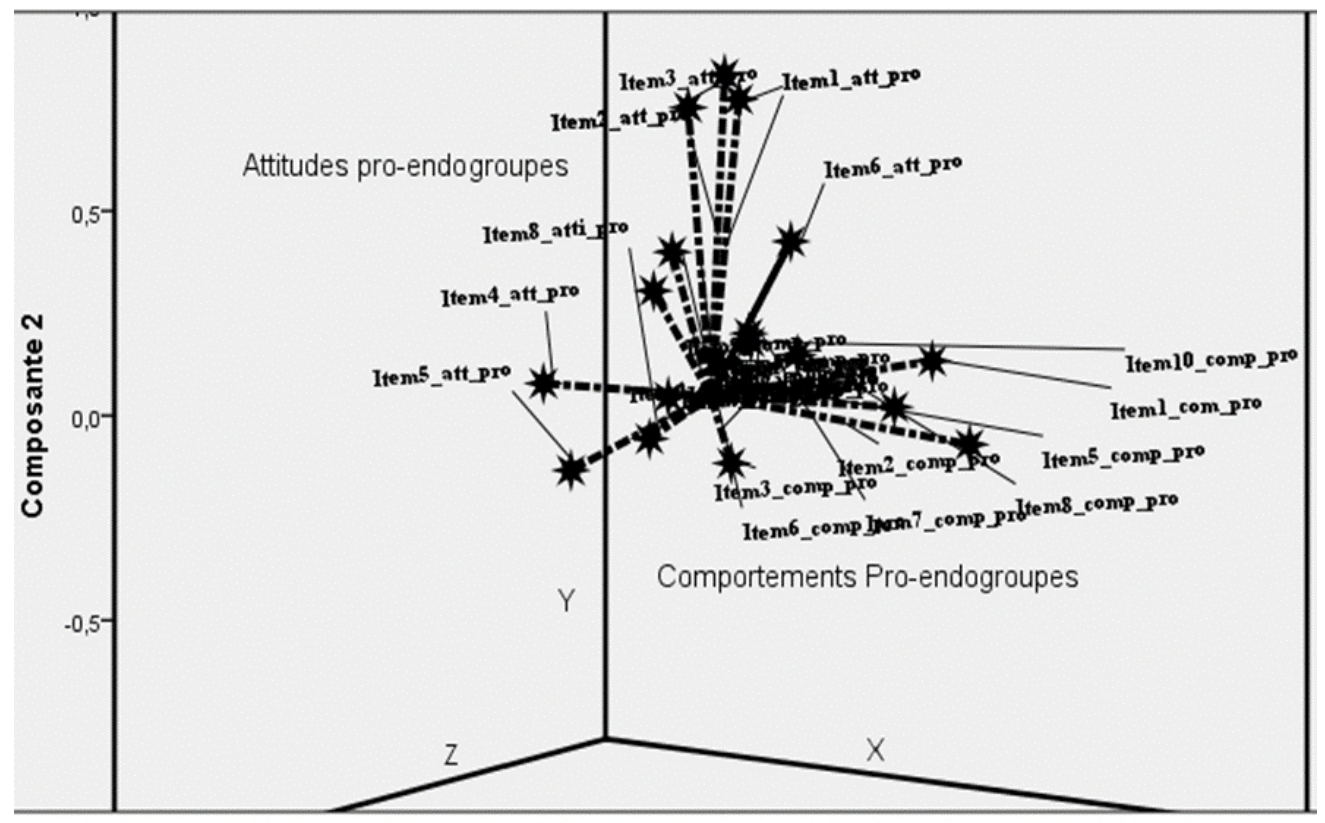

Figure 2: Carte factorielle des dimensions Attitudes et Comportements Pro-endogroupes

Le biais pro-endogroupe est représenté sur ce graphique factoriel distribué sous la forme d'un arbre dont les branches verticales, en majorité, sont les APEn et les branches orientées dans le sens horizontal constituent les CPEn. Cela montre effectivement qu'en majorité, les items développés évaluent le biais pro-endogroupe sur deux dimensions distinctes: les attitudes et comportements favorables à la tribu d'appartenance.

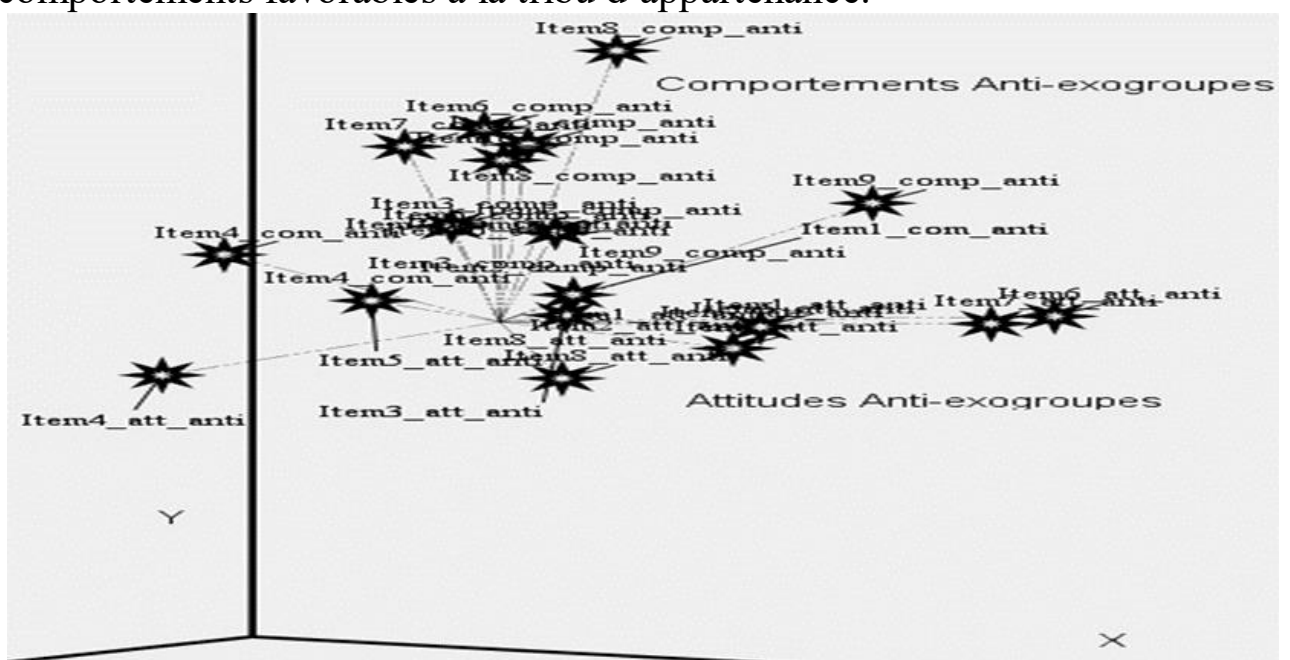

Figure 3: Carte factorielle des dimensions Attitudes et Comportements Anti-exogroupes 
Dans le cas du tribalisme, le biais pro-endogroupe implique une discrimination des autres tribus. Celle-ci se manifeste par des attitudes et des comportements anti-exogroupes. Ce graphique factoriel des points-images montre, en projection, la structure interne de l'exodéfavoritisme en arbre. On y observe une répartition des items relatifs aux AAEx sur l'horizontale et les items des CAEx sur la verticale (partie supérieure de l'arbre). Cela traduit le fait que ces items évaluent à la fois les attitudes et les comportements défavorables aux groupes tribaux de non appartenance. Ainsi, ces différents graphiques montrent que la structure interne de l'échelle du tribalisme présente quatre dimensions distinctes, regroupées en attitudes et comportements pro-endogroupes et anti-exogroupes. Il convient de vérifier si les éléments de cette échelle sont liés les uns aux autres et fournissent un indice de consistance interne satisfaisant.

\section{Fidélité des mesures : Analyse de la consistance interne de l'échelle du tribalisme}

Tableau 2: Statistiques relatives aux indices de consistance interne de l'échelle administrée et de ses sousdimensions, mesures d'association linéaire et variances inter

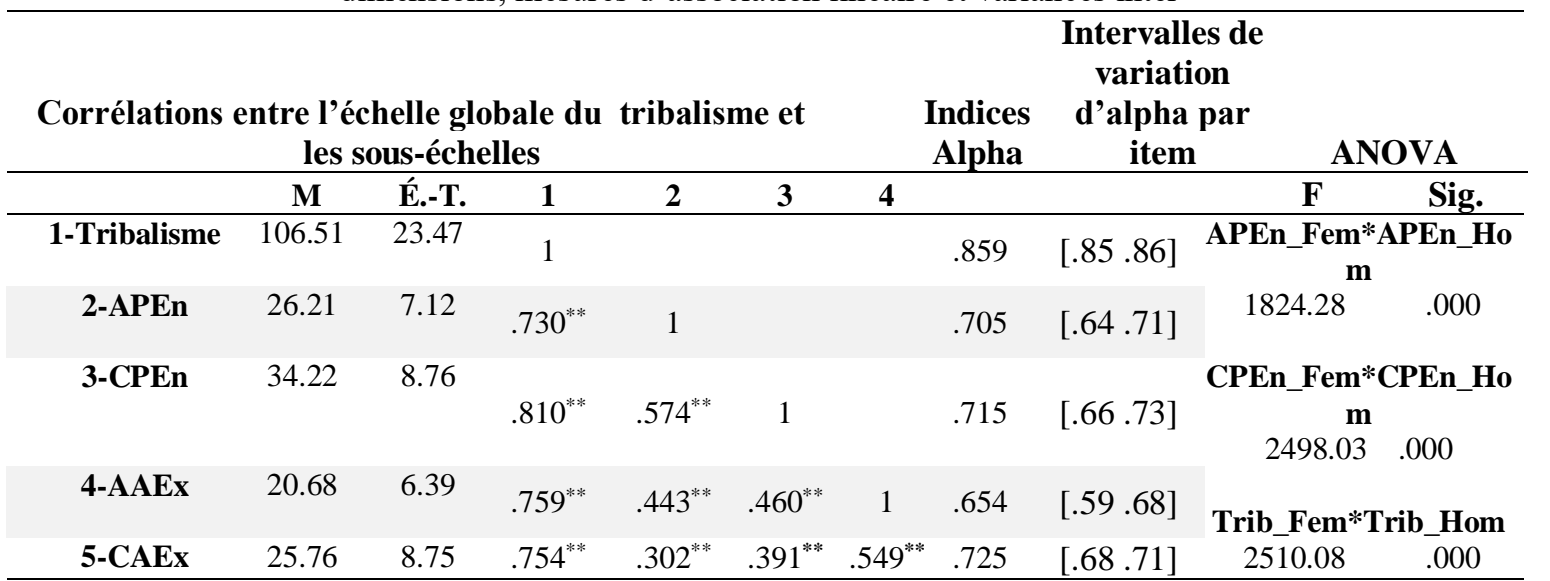

Note : **. La corrélation est significative au niveau .001 (bilatéral)

Au-delà des AFE et des ACP, il est souhaitable qu'à l'intérieur d'un construit, les sous-échelles soient corrélées entre elles (Haccoun, 2013) et que les sous-dimensions du construit soient liées à l'échelle finale (Green et al., 1977). Cette condition permet de valider la dimensionnalité de l'échelle afin de déterminer ses consistances internes. La matrice révèle des corrélations positives et significatives entre le tribalisme et ses dimensions : Tribalisme*APEn $\left(\mathrm{r}=.730^{* *}, p<.001\right)$; Tribalisme*CPEn $\left(\mathrm{r}=.810^{* *}, p<\right.$ $.001)$; Tribalisme*AAEx $\left(\mathrm{r}=.759^{* *}, p<.001\right)$; Tribalisme*CAEx $\left(\mathrm{r}=.754^{* *}\right.$, $p<.001)$ et de bonnes corrélations entre ces dimensions : APEn*CPEn $(\mathrm{r}=$ $\left..574^{* *}, p<.005\right)$; APEn*AAEx $\left(\mathrm{r}=.443^{* *}, p<.001\right)$; APEn*CAEx $(\mathrm{r}$ $\left.=.302^{* *}, p<.001\right)$; CPEn*AAEx $\left(\mathrm{r}=.460^{* *}, p<.001\right)$; CPEn*CAEx $(\mathrm{r}$ 
$\left.=.391^{* *}, p<.001\right) ; \operatorname{AAEx}{ }^{*} \operatorname{CAEx}\left(\mathrm{r}=.549^{* *}, p<.001\right)$. Ces mesures d'association traduisent le degré de liaison du concept du tribalisme avec ses dimensions.

La mesure alpha de Cronbach permet de valider la cohérence interne des échelles psychométriques. Elle ne constitue pas la seule preuve de cette validité. On comble cette lacune en procédant à l'analyse des alphas de Cronbach par item (Bernaud, 2014). La matrice ci-dessus révèle des indices alpha satisfaisants. En effet, les alphas par item de l'échelle du tribalisme se situent dans l'intervalle [.85 .86]. Ceux des sous dimensions APEn, CPEn, AAEx et CAEx sont compris respectivement dans les intervalles [.64 .71] ; [.66 .73]; [.59 .68] et [.68 .71]. Les coefficients alphas de Cronbach de l'échelle finale (.859), et des sous-échelles (Attitudes Pro-endogroupes, $\alpha=$ .705 ; Comportements Pro-endogroupes, $\alpha=.715$; Attitudes Antiexogroupes, $\alpha=.654$ et Comportements Anti-Exogroupes, $\alpha=.725$ ) sont également satisfaisants.

En conclusion, les données statistiques précédentes confortent les observations des graphiques factoriels. Elles montrent que l'échelle psychométrique développée dans la présente étude est fiable, et ses paramètres métrologiques se situent dans les normes requises dans le domaine de l'ingénierie psychométrique (Haccoun, 2013 ; Nunnally, 1978). Pour en éprouver la validité dans les relations intergroupes, on analyse le tribalisme chez les individus en fonction du sexe. Les données collectées permettent de conclure que les femmes ont une tendance beaucoup plus favorable à la tribu d'appartenance que les hommes $(\mathrm{F}(1,890)=1824.287, p<.05))$, tandis que les hommes sont plus exodéfavorables qu'elles $(\mathrm{F}(2,890)=2498.031, p<$ $.05)$. D'un point de vue global, les femmes sont plus tribalistes que les hommes $(\mathrm{F}(3,890)=2510.085, \quad p<.05)$. Ce résultat conforte la validité de l'instrument proposé par la présente recherche, puisqu'un instrument valide est censé mettre en évidence les différences entre deux groupes de répondants pour lesquels des scores distincts sont attendus (Trochim, 2006). Or, on s'attendait notamment, en se basant sur l'hypothèse du mâle guerrier, à ce que les hommes manifestent plus d'attitudes et de comportements xénophobes et ethnocentriques que les femmes (McDonald et al., 2012).

\section{Discussion}

La présente étude avait pour objectif de mettre sur pied une échelle de mesure du tribalisme présentant quatre dimensions relatives aux attitudes et comportements pro-endogroupes (favorables à la tribu d'appartenance) et antiexogroupes (défavorables aux tribus de non appartenance). Elle a consisté en la construction et la validation des propriétés psychométriques de ladite échelle. Les indices métriques calculés sont satisfaisants. Ils attestent de la fiabilité et de la validité de l'échelle. Ces propriétés respectent les normes 
standards dans le domaine de l'ingénierie psychométrique (Roulin, 2017). Dans les détails, au regard de la statistique inférentielle, les poids factoriels calculés sont acceptables. Les analyses factorielles et en composantes principales corroborent la dimensionnalité de cette échelle. Au regard des corrélations linéaires, l'échelle est reliée positivement et significativement à ses dimensions, comme l'exigent les standards en la matière (Haccoun, 2013). De même, la méthode Alpha de Cronbach a fourni des indices satisfaisants. Du point de vue de la validité prédictive, cette échelle distingue bien les groupes de participants et prédit les tendances au tribalisme. Ainsi, elle quantifie et classifie typologiquement les individus auxquels elle est administrée, de façon à déterminer ceux qui présentent les attitudes et comportements pro-endogroupes et anti-exogroupes.

La recherche dans le domaine de la psychométrie définit les paramètres standards de référence qui permettent de vérifier la fiabilité et la validité d'une méthode psychométrique. Ils sont suivis dans la présente étude. En effet, l'échelle du tribalisme développée ici se conforme à la logique des échelles psychométriques ayant un dispositif de réponse. On peut la classer dans la catégorie des tests psychométriques d'auto-évaluation (Ruiller, 2008). Cela exige, après la collecte des données, que l'on définisse obligatoirement les coordonnées métrologiques de l'échelle, en procédant à une Analyse Factorielle Exploratoire (Tay \& Jebb, 2017). Cette méthode statistique a permis d'extraire un ensemble de variables latentes de cet instrument, de manière à restituer le maximum d'informations qu'il comporte (Habib, n.d.). Ces variables sont le résultat des pondérations des pourcentages d'inertie cumulés des facteurs retenus, et dont les coefficients dépendent des poids factoriels fournis par l'échelle. En raison du fait que les données obtenues sont quantitatives et regroupées en facteurs ou en composantes principales, cette procédure statistique s'est appliquée à la validation de l'instrument développé. De fait, elle permet de vérifier si lesdites données forment un contenu factoriel et des dimensions cohérentes. En accord avec cette procédure, l'échelle développée dans la présente recherche comporte une structure interne factorisée suivant les indices KMO connus (Ruiller, 2008).

La littérature psychométrique demande de déterminer les poids factoriels après la vérification de la structure factorielle (Stewart, 1981). Dans cette veine, les pourcentages d'inertie cumulés obtenus constituent une preuve de validité de la structure factorielle de l'échelle du tribalisme. Toutefois, le critère de communalité et de fiabilité des items de cette échelle soutient également les indices des contributions factorielles respectives de l'échelle finale et de ses dimensions ; ces indices sont au-dessus de l'indice standard (.60 ou .70) des échelles psychométriques (Habib, n.d. ; Haccoun, 2013). La méthode d'alpha de Cronbach appliquée apporte des résultats satisfaisants (Bernaud, 2014). De même, le critère de fiabilité de l'échelle a conduit à 
l'établissement du critère de validité prédictive (Trochim, 2006). En effet, la méthode psychométrique développée et validée dans cette recherche permet de différencier les femmes et les hommes, en révélant que les seconds montrent plus d'attitudes et de comportements hostiles à l'exogroupe tribal.

\section{Conclusion}

Cette recherche développe et valide une échelle psychométrique d'évaluation du tribalisme dans les relations intergroupes. En se référant aux normes appliquées dans le domaine de l'ingénierie psychométrique, on peut conclure que cet instrument de mesure est fiable. Il peut, par conséquent, être utilisé pour l'évaluation quantitative du tribalisme, envisagé dans la présente recherche comme une discrimination se manifestant par des attitudes et des comportements favorables à la tribu d'appartenance et défavorables aux tribus de non-appartenance.

\section{References :}

1. Asongu, S. \& Kodila-Tedika, O. (2017). Tribalism and Government Effectiveness. Economics Bulletin, 37, 1, 156-167.

2. Assam, M. (2014). Société tribale kabyle et (re)construction identitaire berbère : le cas des At Zemmenzer (XIXème s.-XXIème) (Thèse de Doctorat). Institut National des Langues et Civilisations Orientales : Paris.

3. Autin, F. (n.d.). La théorie de l'identité sociale de Tajfel et Turner. Laboratoire Savoirs, Cognition et Pratiques Sociales (EA 3815). Université de Poitiers-MSHS.

4. Barth, F. (1995). Les groupes ethniques et leurs frontières. In P. Poutignat \& J. Streiff-Fénart (Éds.), Théories de l'ethnicité. Presses Universitaires de France : Paris.

5. Bernaud, J.-L. (2014). Méthodes de tests et questionnaires en psychologie. Paris : Dunod. Disponible à http://excerpts.numilog.com/books/9782100587957.pdf

6. Berting, J. (2001). Identités collectives et images de l'autre : les pièges de la pensée collectiviste. HERMĖS, 30, 41-58.

7. Bhasin, V. (1991). Status of Women in the Himalayas: A Case of Gaddis. New Delhi : Kamla Raj Enterprises.

8. Churchill, G. A. (1979). A paradigm for developing better measures or marketing constructs. Journal of Marketing Research, 16, 1, 64-73.

9. Dhume, F. (2011). Entre l'école et l'entreprise, la discrimination ethnico-raciale dans les stages. Une sociologie publique de l'ethnicisation des frontières et de l'ordre scolaires (Thèse de Doctorat). Université de Provence : Aix-Marseille I. 
10. Durand, C. (2005). L'analyse factorielle et l'analyse de fidélité. Notes de cours et exemples: Université de Montréal, Département de Sociologie.

https://www.webdepot.umontreal.ca/.../SOCIO/.../notesdecours/analy se_factorielle.pdf

11. Evrard. Y., Pras, B., \& Roux, E. (2000). Market: études et recherche en marketing (2ème Éd.). Paris: Dunod.

12. Fokou Dchoune, F. P., Ebalé Moneze, C., Messanga, G. A., \& Dzuetso Mouafo, A. V. (2012). Effet de la réversibilité du statut de l'endogroupe sur l'exofavoritisme au sein des groupes de statut faible. Journal sur l'identité, les relations interpersonnelles et les relations intergroupes, 5, 17-27.

13. François, P., Rainer, I., \& Trebbi, F. (2014a). « How is Power Shared in Africa? ». Econometrica, forthcoming.

14. François, P., Rainer, I., \& Trebbi, F. (2014b). The Dictator's Inner Circle. NBER Working Paper, 20216.

15. Green, S. B., Lissitz, R. W., \& Mulaik, S. A. (1977). Limitations of coefficient alpha as an index of test unidimensionality. Educational and Psychological Measurement, 37, 827-838.

16. Habib, N. (n. d.). Méthodologie de construction d'une échelle de mesure de la valeur perçue de l'expérience de magasinage : application du paradigme de Churchill. Université d'Auvergne.

17. Haccoun, R. R. (2013). Comprendre et se servir de l'analyse des corrélations : la corrélation et la régression pour les sciences du comportement (Codex PSY6007). Université de Montréal.

18. Henry, P. J. \& Sears, D. O. (2002). The symbolic racism 2000 scale. Political psychology, 23, 253-283.

19. Jacobson, D., \& Deckard, N. (2012). The Tribalism Index: Unlocking the. Relationship between Tribal Patriarchy and. Islamist Militants. New Global Studies, 6, 1, 1-16.

20. Kelly, D.R. (n.d.). Moral Disgust and The Tribal Instincts Hypothesis. To appear in R. Joyce, K. Sterelny \& B. Calcott (Eds.), Signaling, Commitment and

Emotion.

Cambridge, MA: The MIT Press.

21. Kougoum, G. (2009). Pour une église-communauté-de-paix dans un contexte multiethnique conflictuel: Le cas du Cameroun (Thèse de doctorat). Université de Montréal : Canada.

22. Kpotufe, I. (2014). Développement économique de l'Afrique entre tribalisme et régionalisme. Disponible à http://www.imanifrancophone.org/fr/developpement-economique-delafrique-entre-tribalisme-etregionalisme/ 
23. Licata, L. (2007). La théorie de l'identité sociale et la théorie de l'autocatégorisation: le Soi, le groupe et le changement social. Revue électronique de psychologie sociale, 1, 19-33.

24. Lombard, J. (1969). Tribalisme et intégration nationale en Afrique noire. L'Homme et la société, 12, 69-86.

25. Lorcerie, F. (2003). L'école et le défi ethnique. Disponible à $\mathrm{fttp}: / / w w w . e s f-e d i t e u r . f r / d e t a i l / 11 / e c o l e e t-l e-d e f i e t h n i q u e-1-. h t m l f f$

26. Mampouya, J. (1983). Le tribalisme au Congo. Disponible à https://gallica.bnf.fr/ark:/12148/bpt6k3339539h.texteImage

27. Mankou, B. A. (2007). Le tribalisme. Le Portique, 5. Disponible à http://journals.openedition.org/leportique/1404

28. Manna, S., \& Sarkar, R. (2016). The Status of Women in Tribal Society: A Study on three tribal communities in Paschim Midnapore, W.B. Disponible à https://www.researchgate.net/publication/316701874

29. Mbonda, E.-M. (2003). La justice ethnique comme fondement de la paix dans les sociétés pluriethniques. Le cas de l'Afrique. In J. BouladAyoub \& L. Bonneville (Éds.), Souverainetés en crise (pp. 450-500). Les Presses de l'Université Laval : France.

30. Mbouobouo, D. (2018). Le tribalisme, un fléau qui gangrène l'Afrique ? Le Coin Des Membres (Blog). Disponible à http://spacassociation.emonsite.com/blog/le-coin-des-membres/article-le-tribalisme-unfleau-qui-gangrene-l-afrique-par-daouda-mbouobouo.html

31. McDonald, M. M., Navarrete, C. D., \& Van Vugt, M. (2012). Evolution and the psychology of intergroup conflict : The male warrior hypothesis. Philosophical Transactions of The Royal Society B Biological Sciences, 367, 670-679.

32. Messanga, G. A. (2018). Les relations entre l'Etat et la tribu en Afrique : Psychologie sociale des relations intergroupes chez CharlesRobert DIMI. In M. Tsalefac, J. Chatué, \& A. L. Tsala Mbani (dir.), Créativité socio-politique et réécriture de soi en Afrique, Mélanges offerts au Professeur Charles-Robert Dimi (pp. 33-64). Yaoundé : Editions Patrimoine.

33. Minkala-Ntadi, P. (2011). Une phénoménologie du tribalisme dans les sociétés africaines. Disponible à http://pierre-raudhel.overblog.com/article-une-phenomenologie-du-tribalisme-dans-lessocietes-africaines-66572311.html

34. N'Dimina-Mougala, A. (2012). Les conflits identitaires ou ethnopolitiques africains au xxe siècle: caractéristiques et manifestations. Guerres mondiales et conflits contemporains, 248, 4, 97-119. 
35. Nunnally, J. C. (1978). Psychometric Theory. New York, McGraw-Hill Book.

36. Obami, O. (n.d.). Pamphlet contre le tribalisme au Congo. Disponible à https://www.edilivre.com/mag/frontwidget/preview/book/id/768499/

37. Ondoua, A. (2013). Sociologie du corps militaire en Afrique noire : Le cas du Cameroun (Thèse de Doctorat). Université De Rennes 1 : France.

38. Richerson, P. J., \& Boyd, R. (2001). The Evolution of Subjective Commitment to Groups: A Tribal Instincts Hypothesis. In R. Nesse, (ed.), The Evolution and the Capacity for Commitment (pp.186-220). New York, NY: Russell Sage.

39. Roubaud, F. (1995). La question ethnique sur le marché du travail à Yaoundé : discrimination ou solidarité ? Contribution pour le Séminaire préparatoire au Sommet Mondial pour le Développement Social (Copenhague, mars 1995). Disponible à http://horizon.documentation.ird.fr/exl-doc/pleins_textes/pleins_ textes 7/b fdi 57-58/010025078.pdf

40. Roulin, J.-L. (2017). Savoir, Comprendre, Apprendre. Leçons de de psychométrie. Université Savoie Mont Blanc. Disponible à https://www.psychometrie.jlroulin.fr.

41. Ruiller, C. (2008). Le soutien social au travail: conceptualisation, mesure et influence sur l'épuisement professionnel et l'implication organisationnelle : L'étude d'un cas hospitalier (Thèse de Doctorat). Université Rennes 1 : France.

42. Tay, L., \& Jebb, A. T. (2017). Scale Development. In S. Rogelberg (Éd.), The Sage Encyclopedia of Industrial and Organizational Psychology. Thousand Oaks, CA, Sage.

43. Tchatchoua, T. (2012). Les bamiléké au Cameroun: Ostracisme et sous-développement. L'Harmattan : Paris.

44. Trochim, W. M. (2006). The Research Methods Knowledge Base. Disponible à http://www.socialresearchmethods.net $/ \mathrm{kb} /$.

45. Tsafak, G. (2002). Le tribalisme chez les élèves de l'enseignement secondaire au Cameroun. Revue des sciences de l'éducation de MCGILL, 37, 2, 193-222. 


\section{Échelle du Tribalisme}

Consigne : Lisez les affirmations ci-après et indiquez votre degré d'accord ou de désaccord en encerclant le nombre qui correspond à votre opinion. Toutes les réponses que vous donnez seront gardées confidentielles, alors soyez aussi honnêtes que vous le pouvez. 1= $($ Fortement en désaccord $) ; 2=$ (modérément en désaccord $) ; 3=($ Légèrement en désaccord); 4= (neutre) ; 5= (Légèrement en accord); $6=$ (modérément en accord); $7=$ (fortement en accord)

\section{Attitude Pro-Endogroupe}

1 Dans la gestion de ce pays, les problèmes de ma tribu devraient d'abord être résolus avant ceux des autres tribus.

2 Je veux que dans chaque institution, les membres de ma tribu soient majoritaires.

3 Les membres de ma tribu devraient en principe être parmi les plus promus de ce pays.

4 J'aime être en harmonie avec les membres de ma tribu.

5 Ma tribu ne devrait pas être moins valorisée que les autres dans ce pays.

6 Les membres de ma tribu sont plus intelligents que les membres des autres tribus

7 Les membres de ma tribu sont plus respectueux que les autres tribus

8 J'ai de meilleures relations avec les autres membres de ma tribu qu'avec les membres des autres tribus.

\section{Comportement Pro-Endogroupe}

1 Les autres tribus se comportent de manière injuste envers ma tribu.

2 Sans raison apparente, les membres des autres ethnies traitent mal les membres de ma tribu.

3 Le Gouvernement camerounais ne donne pas à ma tribu les avantages qu'elle mérite.

4 Les membres de ma tribu ne devraient en aucun cas être discriminés à l'entrée des grandes écoles de notre pays.

5 Il est normal que les membres de ma tribu soient nommés à des postes de responsabilité dans notre pays.

6 Lors des élections, je vote un candidat en raison du fait qu'il appartient à ma tribu.

7 Chaque fois qu'ils en ont l'occasion, les membres des autres tribus discriminent injustement les membres de ma tribu.

8 L'État devrait faire en sorte que les membres de ma tribu dirigent ce pays.

9 Je suis en mesure de participer à des manifestations pour défendre les intérêts des membres de ma tribu.

10 En ce qui concerne le mariage, il est normal de courtiser et d'épouser essentiellement les femmes de sa tribu.

\section{Attitude Anti-Exogroupe}

1 Dans un pays comme le nôtre, le Gouvernement ne devrait pas résoudre les problèmes des autres tribus avant ceux de ma tribu.

2 Je veux que les membres des autres tribus soient minoritaires dans les institutions de ce pays.

3 Les membres des autres tribus ne devraient pas être les plus promus de ce pays.

4 Je ne veux plus être en harmonie avec les membres des autres tribus.

5 Les autres tribus ne devraient pas être valorisées dans ce pays.

6 Les membres de ma tribu sont plus intelligents que les membres des autres tribus

7 Les membres des autres tribus ne sont pas aussi respectueux que les membres de ma tribu.

8 J'ai de plus bonnes relations avec les membres des autres tribus qu'avec les membres de ma tribu.* Comportement Anti-Exogroupe

1 Les autres tribus se comportent de manière injuste envers ma tribu.

2 Je serai indifférent si un individu appartenant à une autre tribu que la mienne est traité de manière injuste.

3 Le Gouvernement ne devrait pas accorder des avantages aux autres tribus. 
4 Normalement, on ne devrait plus admettre les membres des autres tribus dans les grandes écoles de ce pays.

5 Il serait normal que l'État réduise les postes de responsabilité attribués aux membres des autres tribus.

6 Pendant les élections, je ne voterai plus pour les candidats qui ne sont pas de ma tribu.

7 Chaque fois qu'il y en aura l'occasion, j'approuve l'idée que les membres des autres tribus soient insultés ou agressés.

8 On devrait faire en sorte que ce pays ne soit plus dirigé par les membres d'autres tribus que la mienne.

9 Je suis en mesure de participer aux manifestations contre les membres des autres tribus qui igent ce pays.

10 En ce qui concerne le mariage, il n'est pas normal de courtiser et d'épouser les femmes des autres tribus. 